\title{
Eine Erweiterung des Dopplerschen Prinzips in der Astrophysik.
}

In A. N. 4749 ist für meine Erklärung eines Ringnebels, als seitlich gesehenen Wirbelringes, auf $\mathrm{S} .406$ als zweiter Grund angeführt, daß die Radialgeschwindigkeit für eine Verstärkung der Lichteindrücke durch Schaffung einer Komponente nach dem Beșchauer hin sorgt.

Diese These hat auf dem Gebiete der Schallschwingungen in neuester Zeit gewichtige Bestätigungen erhalten. Nach $F$. Auerbach und Mach hat sich bei artilleristischen Schießversuchen herausgestellt, daß dann, wenn die Geschoßgeschwindigkeit der Fortpflanzungsgeschwindigkeit des Schalles ubberlegen ist, ein Schuß in der eigentlichen Schußrichtung früher vernommen wird, als in anderen Richtungen $(F$. Auerbach: Physik des Krieges. Jena I 9 I 5 bei G. Fischer). Diese frühere Vernehmbarkeit darf ohne weiteres als ein Ausdruck stärkerer Hörbarkeit, also größerer Intensität der Schallwirkung, angesehen werden. $\mathrm{Daß}$ dem wirklich so ist, dafür scheinen ferner einige Beobachtungen im gegenwärtigen Kriege zu sprechen. Es sind folgende:

Die Kanonaden auf Dünkirchen I 9 I 5 Mai 6 und bei der Einnahme von Libau I 9 I 5 Mai 8 wirkten in der Schußrichtung der schweren Landgeschütze, dort auf etwa $115 \mathrm{~km}$, hier sogar auf $206 \mathrm{~km}$ Entfernung, mit dem Erfolge, daß Gebäude erbebten und Fensterscheiben zerbrachen. Jenes wurde unter Mai 8 aus Dover, dieses aus Wisby auf Gotland berichtet. Die Grenzen der "Zone des Schweigens", die zwischen dem Umkreis direkter Hörbarkeit und der Zone des hochatmosphärischen Echos liegt (nach von dem Borne), wurden bei der Beschießung Antwerpens, I 9 I 4 Okt. 7-9., nach E. v. Everdingen, im Gebiete des Meteorologischen Dienstes der Niederlande, auf $100 \mathrm{~km}$ innen und $160 \mathrm{~km}$ außen vorgeschoben. Das war in der Schußrichtung der deutschen schweren Batterien, die der direkten Hörbarkeit demnach eine Reichweite von $100 \mathrm{~km}$, anstatt sonst 30 bis höchstens $60 \mathrm{~km}$, verschaffte.

Beim Lichte handelt es sich allerdings nicht, wie beim Schall, um Longitudinalwellen, sondern um Transversalwellen, deren Wirkungsstärke nach den zur Zeit geltenden Anschauungen abhängt von einer Amplitude senkrecht zur Strahlungsrichtung, einer Amplitude, die deshalb an einer Komponente in dieser Richtung gänzlich unbeteiligt bleibt. Dieser Einwand, auf den mich der Assistent am Physikalischen Institut zu Kiel Dr. Hans Schmidt gesprächsweise aufmerksam machte, kann schon im Rahmen jener geltenden Anschauungen ausgeschlossen werden durch folgende Uberlegung.

Bei dem Übergang der Lichtschwingungen vom leuchtenden Ringnebel zum Äther bewirkt jedes radial auf diesen gerichtete Teilchen der lichtspendenden Masse des Wirbelringes eine örtliche Verdichtung des Äthers. Jedes radial von ihm fortstrebende Teilchen bewirkt eine örtliche Verdünnung. Jenes wirkt pressend, dieses wirkt saugend. Von einer verdicbteten Ätherpartie sind aber wuchtigere Schwingungen zu erwarten, als von einer verdünnten. So wird dort, wo die radial auf den Beschauer gerichtete Schar leuchtender Teilchen einfällt, eine Front stärkerer Lichtwirkung bemerkbar sein. Dort, wo die radial vom Beschauer fortstrebende Schar sich geltend macht, wird eine Zone schwächerer Lichtwirkung entstehen, die, an der Grenze der Sichtbarkeit, zu vollständiger Auslöschung führen kann.

In dem gemeinsamen Ergebnis aller dieser Überlegungen darf eine Weiterführung des Dopplerschen Prinzipes erkannt werden, da dieses selbst schon auf eine Verstärkung der chemischen Wirkung des Lichtes in der radial auf den Beobachter gerichteten Fortpflanzung, auf ihre Schwächung in entgegengesetzter Richtung hinauslief. Auch die volle Erkenntnis dieses Prinzips geschah zuerst an Schallerscheinungen. Sehr liegt, im Interesse dieses wissenschaftlichen Fortschrittes, an einer Vervollständigung der eingangs erwähnten Detonations-Beobachtungen auf den gesamten Umkreis ihrer Schallquellen. Das kann vor allem auch durch genaue Festlegung solcher früheren Beobachtungen an den erwähnten Tagen geschehen.

Schnelsen, I 9 I 5 Mai 13.
Wilhelm Krebs.

\section{Helligkeit und Gestalt des Kometen $1913 \mathrm{f}$ (Delavan).}

Gesamthelligkeit und Schweiflänge des Kometen wurden mit bloßem Auge geschätzt, Schweifrichtung (durch Einweisen) und andere Details mit einem Prismenglas $\mathrm{O}=50 \mathrm{~mm}, \mathrm{~V}=\mathrm{r} 0$. Die Extinktion ist streng gerechnet und angebracht. Der Stufenwert fand sich auffallend klein; aus drei verschiedenen Bestimmungen kam der Reihe nach $0^{\mathrm{m}} .3^{\circ} 0^{\circ}$ $0 \mathrm{~m} .024,0^{\mathrm{m}} .023$, im Mittel $0^{\mathrm{m}} \circ 2_{6} 6$ heraus, abgeleitet mit den Potsdamer Helligkeiten der Vergleichsterne.

\begin{tabular}{|c|c|c|c|c|c|c|c|c|c|c|c|}
\hline IOIA & M. Z. & $\begin{array}{l}\text { Beob. } \\
\text { Hellig- }\end{array}$ & $Z D \not$ & Vergleichsterne & $\begin{array}{l}\text { Red. auf } \\
r=I\end{array}$ & \begin{tabular}{|l|} 
Reduz. \\
Hellig-
\end{tabular} & & & Schwei & & Bemerkungen \\
\hline 1914 & Straßb. & keit & & & & & Länge & P.-W; & $t_{\odot}$ & $p-p_{\odot}-\mathrm{I} 80^{\circ}$ & \\
\hline $\begin{array}{ll}\text { Okt. } & \text { I I } \\
& \text { I } 2\end{array}$ & $\left|\begin{array}{cc}7^{\mathrm{h}} & 5^{6^{\mathrm{m}}} \\
7 & 16\end{array}\right|$ & $\begin{array}{l}3^{\mathrm{m}} \cdot 02 \\
3.10\end{array}$ & $\begin{array}{l}80^{\circ} \\
75\end{array}$ & $\mid \begin{array}{c}\text { I } 2 \alpha \text { Can. ven., } \gamma \text { Boot. } \\
》\end{array} \delta$ Urs.ma. & $\begin{array}{l}-\mathrm{Im}^{\mathrm{m}} \mathrm{.} 8 \\
-\mathrm{I.27}\end{array}$ & $\begin{array}{l}\mathrm{m} \cdot 74 \\
1.83\end{array}$ & $\begin{array}{l}5 \cdot 5 \\
7\end{array}$ & $3.3^{\circ}$ & $179^{\circ}$ & $-27^{\circ}$ & Heller scharfer Kern \\
\hline
\end{tabular}

Anzeige. Von der in Nr. 4792 zum Abdruck gebrachten »Tafel zur Berechnung der Präzession für polnahe Sterne gültig für 1900.0 und für die Newcombsche Präzessionskonstante " ist eine kleine Anzahl Separatabzüge bei der Redaktion vorhanden. Dieselben werden zum Preise von je r.zo . H. abgegeben.

Berichtigung zu Nr. 478 I Bd. 200 p. 75 Z. I9 v. u. statt 746 [1913 QZ] lies 747 [19I3 QZ].

* $\leadsto 4794$ Bd. 200 p. 309. Die ersten Zifferngruppen der letzten beiden Zeilen sind statt 792, I7 I5 zu lesen 772, 1915.

" $" 4803$ Bd. 201 p. 51 Fußnote 2. Der Titel der hier erwähnten Arbeit von cle Sitter lautet: Remarks on Mr. U'oltjer's paper concerning Seeliger's hypothesis. Kon. Akad. van Wetensch. Ie Amsterdam I9 4 , Juli 3.

ఎ 4803 Bd. 201 p. 53 Fubnote 1 statt Luftdruck lies Lichtdruck.

Inhalt zu Nr. 4805. A. Wilkens. Über die Säkulargleichungen der vier helleren Jupitermonde. 85. - W. Krobs. Eine Erweiterung des Dopplerschen Prinzips in der Astrophysik. 107. - C. Wirtz. Helligkeit und Gestalt des Kopneten I913 f (Delavan). 107. Anzeige. 107. - Berichtigungen. 107.

Geschlossen rgis Juli 2. Herausgeber: H. Kobold. Druck von C. Schaidt. Expedition: Kiel, Moltkestr. 80. 